\title{
Pros and Cons of Portal Vein Embolization With Hematopoietic Stem Cells Application in Colorectal Liver Metastases Surgery
}

\author{
VLADISLAV TRESKA ${ }^{1}$, JAN BRUHA ${ }^{1}$, VACLAV LISKA ${ }^{1}$, JAKUB FICHTL ${ }^{1}$, \\ KRISTYNA PROCHAZKOVA ${ }^{1}$, TEREZA PETRAKOVA ${ }^{1}$ and PETR HOSEK ${ }^{2}$ \\ ${ }^{1}$ Department of Surgery, School of Medicine, University Hospital in Pilsen, Pilsen, Czech Republic; \\ ${ }^{2}$ Biomedical Centre, School of Medicine, Pilsen, Czech Republic
}

\begin{abstract}
Background/Aim: Portal vein embolization (PVE) with autologous stem cells application (aHSC) is a method for future liver remnant volume (FLRV) increase. The aim of the study was to evaluate the positivite and negativite aspects of the method in clinical practice. Patients and Methods: PVE with aHSC application was used in 32 patients with colorectal liver metastases and insufficient FLRV. Preoperative number of colorectal liver metastases (CLMS) was 5.2 \pm 3.6 , CLMs volume $70.1 \pm 102.3 \mathrm{~mm}^{3}$. Results: FLRV growth occurred after 2-3 weeks in 31 (96.9\%) patients, with volume increase from $528.2 \pm 170.5$ to $715.4 \pm 143.3 \mathrm{ml}$ $(p=0.0001)$. Postoperative thirty days mortality, morbidity was $0 \%$ and $3.1 \%$, respectively. Insufficient FLRV growth occurred in one patient. RO liver resection was performed in $27(87.1 \%)$ patients. CLMs volume progression was in 5 (15.6\%) patients from $680.0 \pm 59.4$ to $723.1 \pm 57.1 \mathrm{ml}(p=0.01)$. One and two-year overall survival were $88 \%$ and $62.9 \%$ respectively. Six and twelve-month recurrence-free survival rates were $50.7 \%$ and $39.6 \%$ respectively. Conclusion: PVE with aHSC application is a safe and useful method for FLRV growth. It significantly increases secondary CLMs resectability. However, it can cause CLMs progression. Liver resection should, therefore, be performed as soon as possible after achieving optimal increase of FLRV.
\end{abstract}

Colorectal cancer (CRC) ranks among the three most frequent malignant tumours worldwide. Its incidence is approximately 1.3 million cases annually and over 600,000

This article is freely accessible online.

Correspondence to: Vladislav Treska, Professor of Surgery, Head of the Department of Surgery, University Hospital, Alej Svobody 80, 30460 Pilsen, Czech Republic. Tel: +42 03771042701, e-mail: treska@fnplzen.cz

Key Words: Colorectal liver metastases, future liver remnant volume, portal vein embolization, stem cells, tumour stimulation. patients die of this disease over the same period of time (13 ). Colorectal liver metastases (CLMs) are diagnosed concurrently with the primary tumour (synchronous metastases) in 20-25\% of patients and metachronous metastases develop in $40-50 \%$ of patients after surgery for the primary CRC at various intervals of time. Liver resection remains the only radical treatment modality that significantly prolongs patient overall survival. Unfortunately, liver resection is feasible in only $20-30 \%$ of patients. The main cause of non-resectability is the insufficient future liver remnant volume (FLRV) $(4,5)$. Several methods that increase the insufficient FLRV and thus enable secondary resectability of CLMs exist. These include portal vein embolization (PVE) on the CLMs side with application of autologous hematopoietic stem cells (aHSC) into the contralateral branch of the portal vein. We have been using this method for ten years now.

The aim of the study was to evaluate PVE with aHSC application from the point of its positive (FLRV growth stimulation) as well as possibly negative aspects (tumour growth stimulation).

\section{Patients and Methods}

The Ethics committee approval was received for this study from Institutional Ethics Committee (decision date 12/8/2014, No $326 / 2014$ ). We obtained the written informed consent from all patients who participated in this study. In a prospective study, we used this method from June 2010 to April 2020 in 32 patients with insufficient FLRV to increase secondary resectability of CLMs. In the same period, we performed liver surgery for CLMs in 568 patients. Patient enrolment in the treatment was decided by a multi-disciplinary team. The indication for PVE with the application of aHSC was an insufficient FLRV of $<30 \%$ in patients with healthy liver tissue and of $<40 \%$ in patients with liver steatosis and steatofibrosis or, mainly, in patients who had undergone neoadjuvant systemic therapy. Contraindications included the presence of extrahepatic metastases demonstrated using hybrid methods - positron emission tomography or positron emission magnetic resonance imaging. Serious polymorbidity of patients was another contraindication. The average age of the 


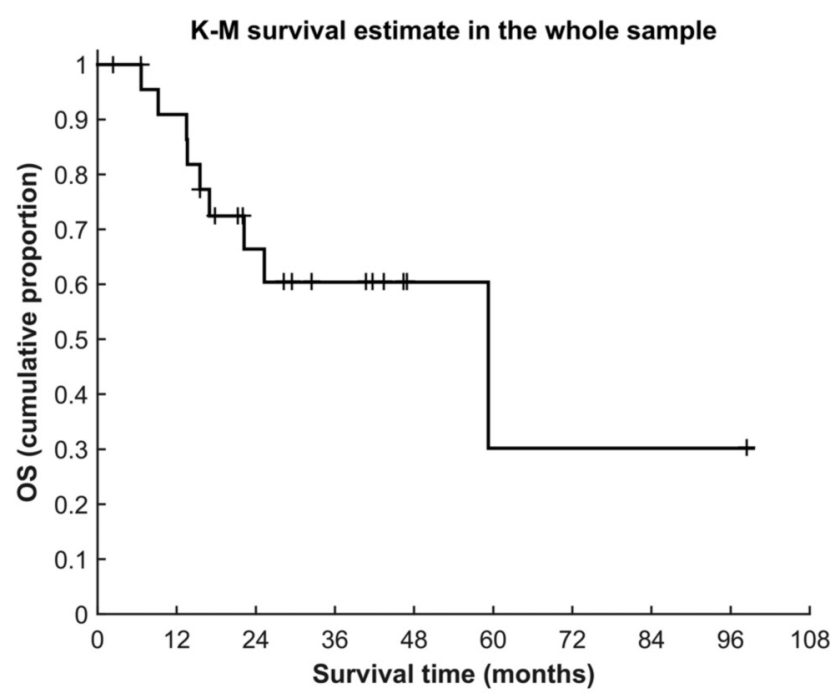

Figure 1. Overall survival of the whole group $(N=32)$. A Kaplan-Meier estimate of the survival function for OS in the whole sample of patients.

patients was 52.6 years (44-73) and the male to female ratio was $2: 1$. The average number of CLMs was $5.2 \pm 3.6$ and their volume was $70.1 \pm 102.3 \mathrm{ml}$. In $8(25.8 \%)$ patients, the CLMs involved both liver lobes, which is why we first "cleared" the left liver lobe using metastasectomy or radiofrequency ablation before undertaking PVE with aHSC application. We used aHSC in the first 15 patients from blood and in the subsequent 17 cases from bone marrow, as the latter methodology was simpler and consisted of aHSC collection and application during one stage operation (Table I). We have described both methods in detail in our previous publication (6). Growth of the contralateral liver lobe was monitored in the case of both methods using CT liver volumetry with manual segmentation (Somatom Definition Flash, Syngo Volume, Siemens) at weekly intervals until optimum FLRV growth occurred. Median follow-up time was 31.8 months.

Standard frequency tables and descriptive statistics were used to characterize the patient group. Recurrence-free survival (RFS) was determined from the date of surgery to the date of the first documented disease recurrence or death. Overall survival (OS) was determined from the date of surgery to the date of death, regardless of its cause. Patients who had not progressed or died were censored at the date of last follow-up. RFS and OS functions observed in the whole sample were estimated using the Kaplan-Meier method. Median survival times and observed proportions surviving at given time points were calculated from the Kaplan-Meier estimates of survival functions using linear interpolation between the nearest complete observations. Median follow-up was estimated from OS data using the inverse Kaplan-Meier method. Associations of the number and volume of CLMs, with RFS and OS were assessed using univariable Cox proportional hazards model. In order to visualize these associations and detect possible non-proportional effects, the results were reviewed using automated stratification. In this procedure, the best-performing threshold (cut-off) value of the independent variable was determined by an automated optimization process finding the threshold providing the lowest Log rank $\mathrm{p}$ value in two-sample Kaplan-Meier analysis. All reported $p$-values are two-

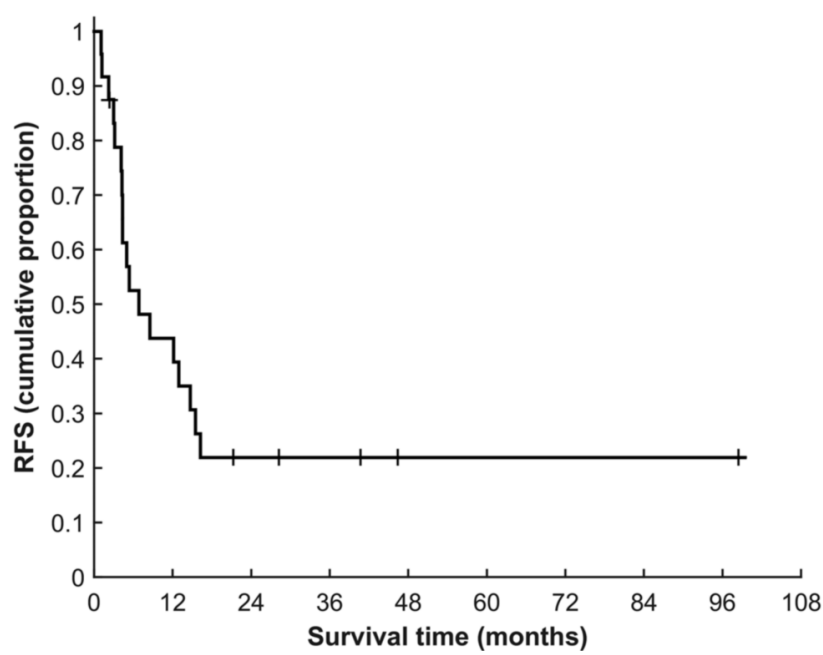

Figure 2. Recurrence-free survival of the whole group $(N=32)$. A Kaplan-Meier estimate of the survival function for RFS in the whole sample of patients. tailed and the level of statistical significance was set at $\alpha=0.05$. Statistical processing and testing was performed in STATISTICA data analysis software system (StatSoft, Inc.2013, Version 12, www.statsoft.com) and Matlab (2019b, MathWorks Inc., Natick, MA, USA).

\section{Results}

Optimal FLRV growth occurred at an interval of 2-3 weeks in $31(96.9 \%)$ patients, with an increase from $528.2 \pm 170.5$ to $715.4 \pm 143.3 \mathrm{ml}(p=0.0001)$. No patient died within 30 days following PVE and aHSC application. Insufficient FLVR growth occurred in one patient $(3.1 \%)$. In one patient $(3.1 \%)$ there was partial leakage of the embolization material into the left lobe during PVE of the right branch; but this did not lead to any clinical symptoms or laboratory alterations. We performed R0 liver resection in $27(87.1 \%)$ patients: 18 right and 9 extended right hepatectomy with zero 30-day postoperative mortality. Grade II-III complications according to the Clavien-Dindo classification occurred in 12 (37.5\%) patients (four cases of biliary leak, five of fluid next to the resection surface, one trauma to the biliary tract, one bleeding from the resection surface, one liver insufficiency). Adjuvant systemic oncological therapy was used in 18 $(66.7 \%)$ of the 27 operated patients. It was not possible to perform liver resection in $5(15.6 \%)$ patients. The reasons included CLMs progression in $5(15.6 \%)$ patients from $680.0 \pm 59.4$ to $723.1 \pm 57.1 \mathrm{ml}(p=0.01)$. However, these patients also had sufficient FLRV growth. The 1- and 2-year OS were $88 \%$ and $62.9 \%$ respectively (Figure 1). Six and twelve-month RFS were $50.7 \%$ and $39.6 \%$ respectively (Figure 2). The number of CLMs had a greater impact on 

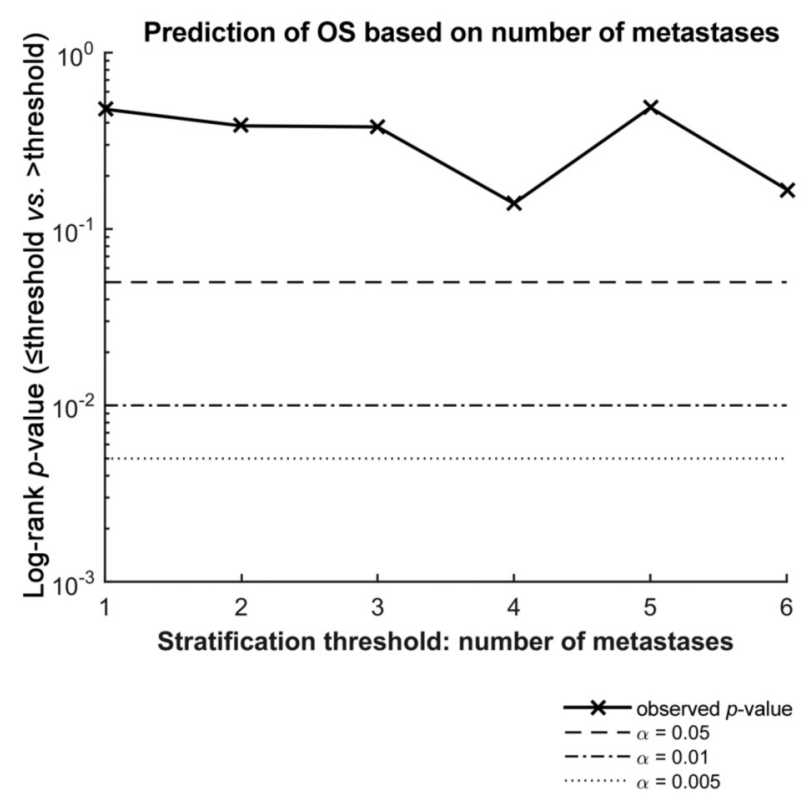

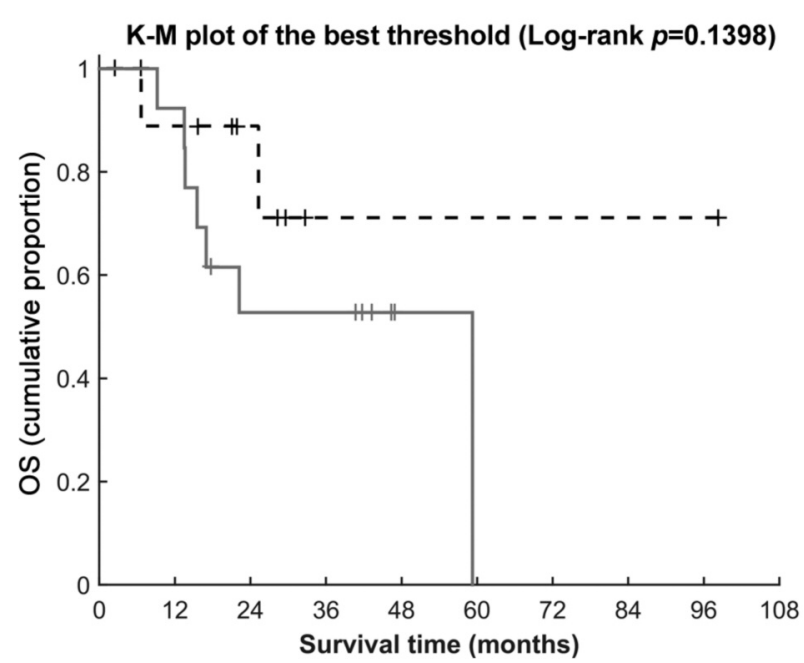

- - number of metastases $\leq 4(n=11)$ number of metastases $>4(n=13)$

Figure 3. Stratification analysis of the association between number of metastases and OS. Left: Log rank p-values for comparison of OS between groups of patients divided according to different threshold values of the metastases number. Right: Kaplan-Meier survival functions for groups of patients defined according to the best-performing threshold value (i.e. at the lowest point of the left-side chart).

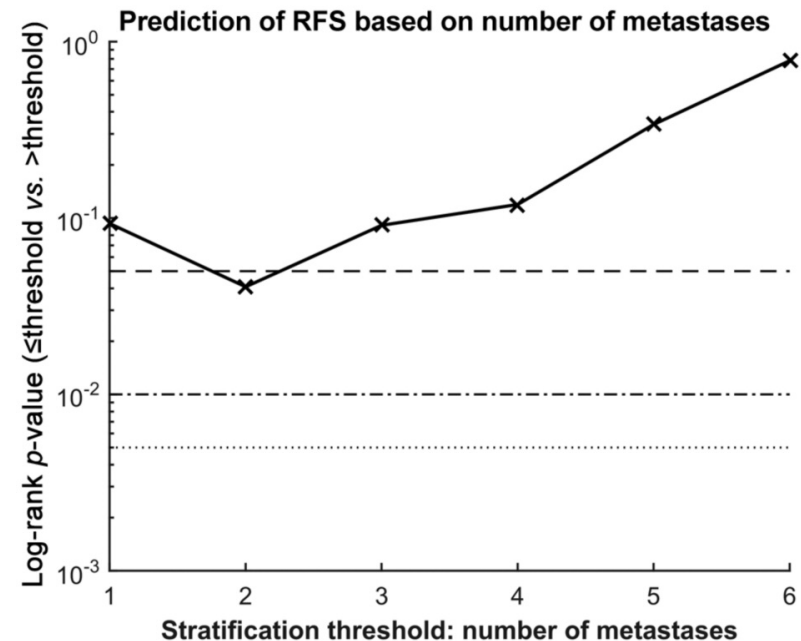

Stratification threshold: number of metastases

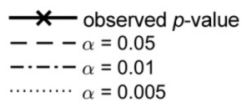

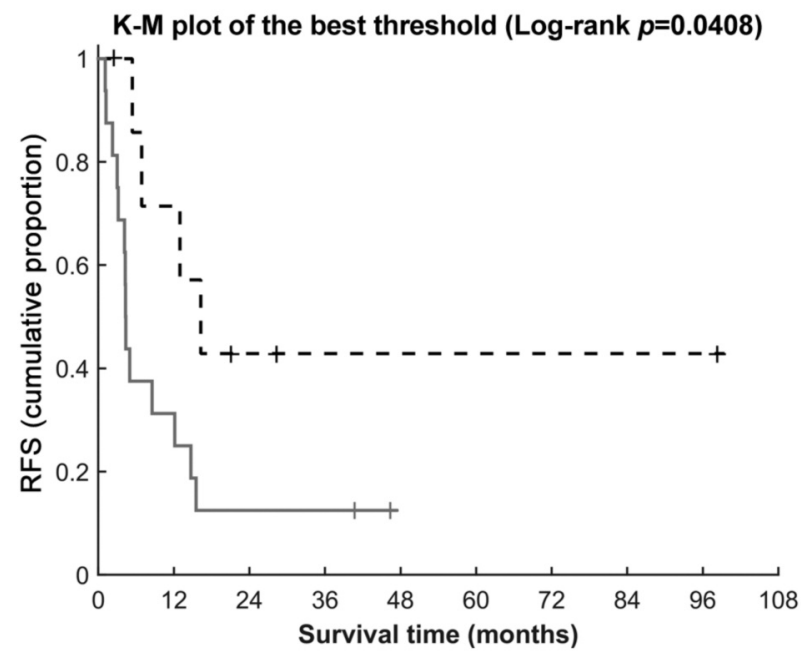

- - number of metastases $\leq 2(n=8)$ number of metastases $>2(n=16)$

Figure 4. Stratification analysis of the association between number of metastases and RFS. Left: Log rank p-values for comparison of RFS between groups of patients divided according to different threshold values of the metastases number. Right: Kaplan-Meier survival functions for groups of patients defined according to the best-performing threshold value (i.e. at the lowest point of the left-side chart).

RFS ( $p=0.04)$ than on OS ( $p=0.14)$ - Figures 3 and 4. The prognostic significance of CLMs volume was highly variable, depending on the selected threshold (Figures 5 and 6). This was due to the small sample size.

\section{Discussion}

The prognosis of patients with CLMs who cannot undergo radical R0 liver resection is dismal despite significant 

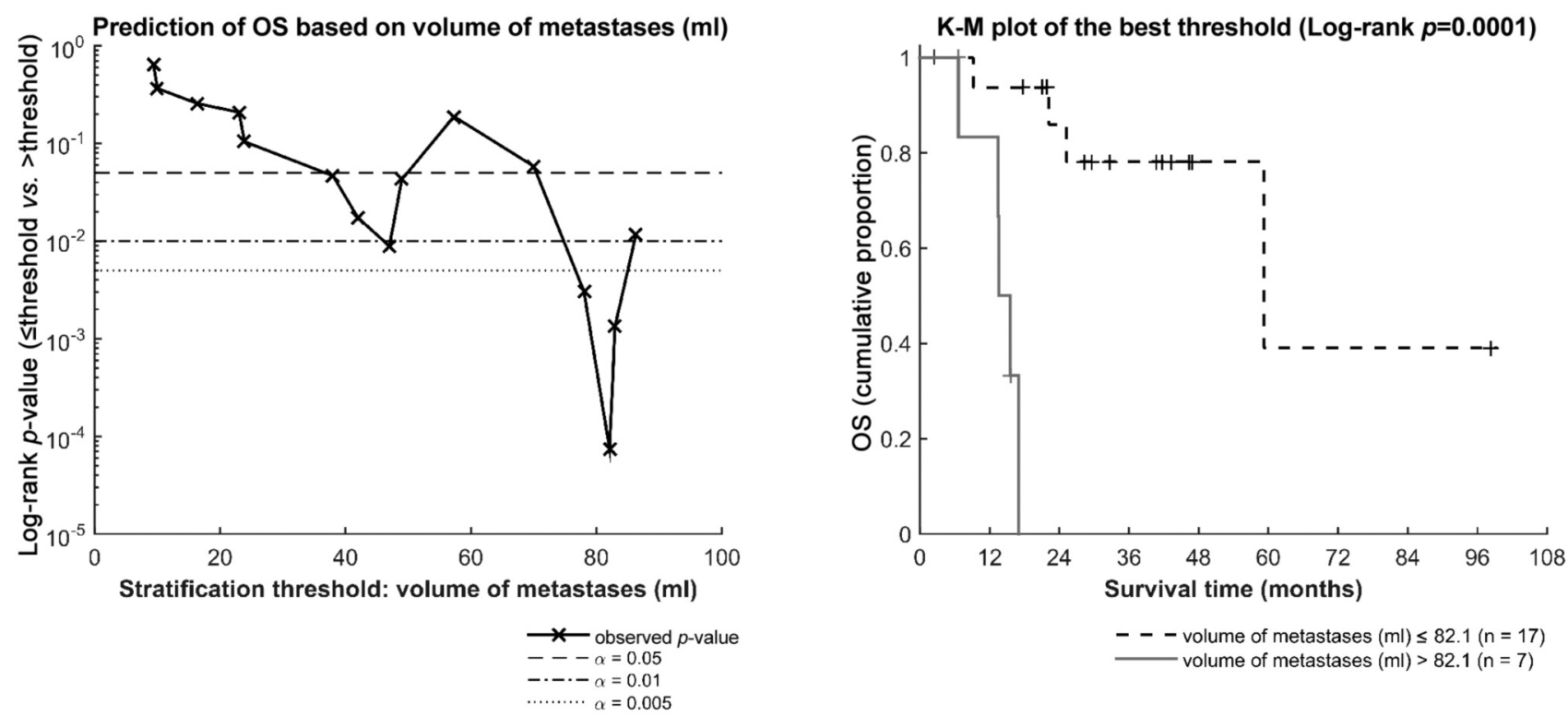

- - - volume of metastases $(\mathrm{ml}) \leq 82.1(\mathrm{n}=17)$

volume of metastases $(m l)>82.1(n=7)$

Figure 5. Stratification analysis of the association between volume of metastases and OS. Left: Log rank p-values for comparison of OS between groups of patients divided according to different threshold values of the metastases volume. Right: Kaplan-Meier survival functions for groups of patients defined according to the best-performing threshold value (i.e. at the lowest point of the left-side chart).
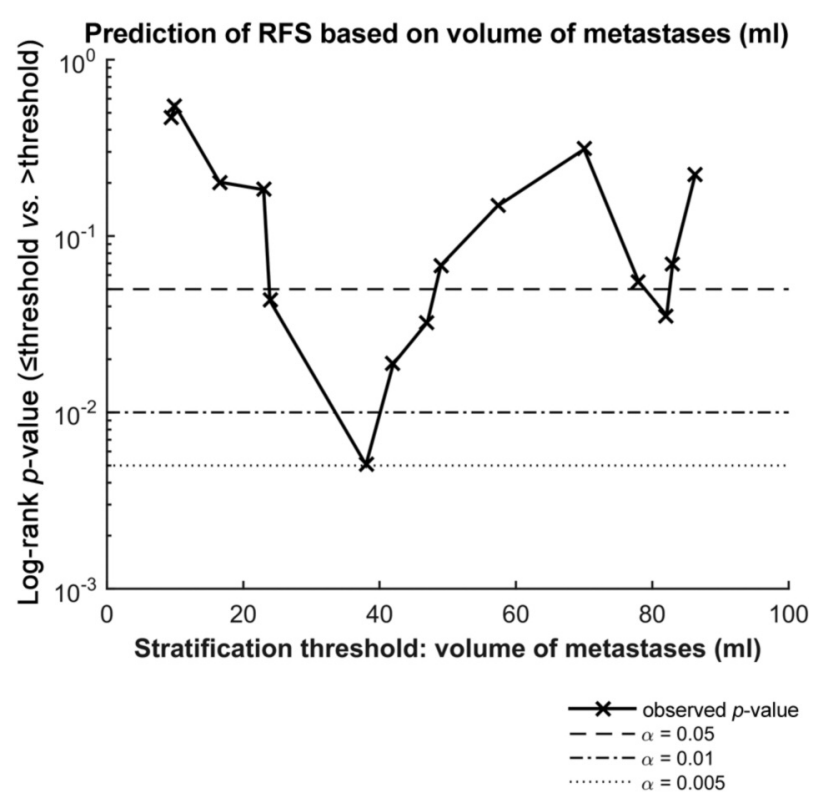

K-M plot of the best threshold (Log-rank $p=0.0050$ )

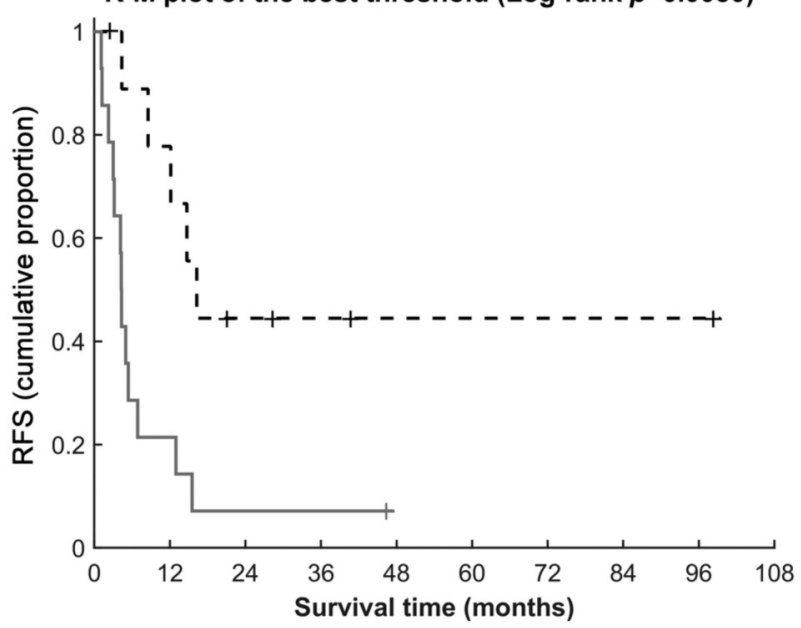

- - - volume of metastases $(\mathrm{ml}) \leq 38(n=10)$ volume of metastases $(\mathrm{ml})>38(n=14)$

Figure 6. Stratification analysis of the association between volume of metastases and RFS. Left: Log rank p-values for comparison of RFS between groups of patients divided according to different threshold values of the metastases volume. Right: Kaplan-Meier survival functions for groups of patients defined according to the best-performing threshold value (i.e. at the lowest point of the left-side chart).

progress in systemic oncological therapy, with less than $20 \%$ of patients remaining alive at 3 years (6). The main cause of CLMs non-resectability is an insufficient FLRV to meet the organism's metabolic needs after surgery. Several methods that increase insufficient FLRV and thus enable secondary resectability of CLMs exist (7-10). Nonetheless, the success rate of certain methods such as PVE, portal vein ligation or staged liver resection from the aspect of FLRV growth is 
Table I. Clinical and demographic characteristics of the study population.

\begin{tabular}{lc}
\hline Demographic data of patients & \\
Patients & 32 \\
Age & $52.6(44-73)$ years \\
Men to female ratio & $2: 1$ \\
Time interval & $6 / 2010-4 / 2020$ \\
Median follow-up time & 31.8 months \\
CLMs characteristics & $5.2 \pm 3.6$ \\
Number & $70.1 \pm 102.3 \mathrm{~mm}^{3}$ \\
Volume & $8(25.8 \%)$ patients \\
Both liver lobes involved & \\
aHSC source & $15(46.9 \%)$ patients \\
Blood & $17(53.1 \%)$ patients \\
Bone marrow & \\
\hline
\end{tabular}

only around $60 \%$. Also, the time needed to achieve optimum FLRV growth in the case of these methods is 4-8 weeks, i.e. relatively long, increasing the risk of CLMs progression or tumour dissemination (11-13). This is why another technique was introduced - Associating Liver Partition and Portal Vein Ligation for Staged hepatectomy (ALPPS), which utilizes a two-stage procedure with maximum reduction of the time interval between both procedures, which is fundamental for reducing the growth of the tumour in the liver and its extrahepatic spread. The first procedure is ligation of the right portal vein and portal branches to segment four on the side of the CLMs, whilst leaving the supply of the liver by arterial blood, and the in situ dissection of the liver parenchyma between the lateral and medial sectors of the left hemi-liver. This method achieves FLRV growth of 40-80\% at an interval of 6-9 days after the first stage of the procedure or up to a $22 \%$ daily increase in FLRV $(14,15)$. However, an enduring problem of this method is the associated high morbidity (around 35\%) and mortality (around 8\%). Other method includes Liver Venous Deprivation, where optimum FLRV growth occurs in 14 days. This is a new method that has not as yet been tested and verified on a larger sample of patients (16-18).

We opted for PVE with aHSC application following previous experimental tests in animals as it is economically viable and safe for patients. One of its undeniable advantages is its simplicity, whereby we currently use a one-stage procedure, i.e. aHSC collection and application during onestage surgery. Another advantage is the relatively fast rate of FLRV growth, achieving optimum parameters over 2-3 weeks in more than $90 \%$ of patients, which significantly increases the secondary resectability of CLMs and substantially prolongs patient OS.

The disadvantage of this method is the increase in CLMs volume, which occurred in $15.6 \%$ of our patients and which then caused non-resectability of the CLMs. This method involves two mechanisms (PVE and aHSC application) that stimulate liver parenchyma growth and regeneration via cytokines and growth factors, which may also presumably stimulate cancer cell growth. The question remains whether interaction of aHSC with cancer cells may also play a role in CLMs progression, which could be induced by active remodelling of aHSC into cancer cells or induction of a stromal cell environment advantageous to the CLMs from the aspect of chemoresistance, inhibition of apoptosis and promotion of cancer cell growth. In our previous studies $(19,20)$ we demonstrated that PVE with aHSC application stimulates faster CLMs growth compared to PVE, even though the subsequent increase in CLMs volume over the given interval of time did not differ statistically significantly between both methods. At this time, it is unclear to what degree both methods are involved in CLMs growth. Our current results show a trend towards more rapid CLMs progression and subsequent worse RFS and OS in patients with an overall greater number and larger volume of CLMs, although we were unable to demonstrate an unequivocal statistical significance of either of these factors given the size of our sample. The biological activity and location of the primary tumour also undoubtedly plays a role (21-23). Further clinical and experimental research is needed to address these issues. At this time, liver resection performed as soon as possible once optimum FLRV growth has been achieved without further delay that could lead to cancer cell growth and dissemination is essential in clinical practice. In this sense, the question of chemotherapy or target therapy during the period of FLRV growth arises. This approach could be beneficial in preventing the growth of CLMs and could thus increase the efficiency of the method. Further clinical and experimental research is needed to address these issues. We are aware of a number of limitations that could affect the results of the study. These mainly include the small number of patients included up to now; the heterogeneity due to using two groups of patients with aHSC application as well as the differences in the biological activity and location of the primary tumour. Also, the biological activity of CLMs in a given patient will be undoubtedly significant from the aspect of long-term results. This is why we are currently continuing our clinical research with the aim of answering the questions raised above.

\section{Conclusion}

PVE with aHSC is the method of choice in patients suffering from CLMs and insufficient FLRV. Its advantage lies with the fact that it is one-stage simple procedure and has very low complications rates. It is a method which can be chosen as one-stage procedure in patients suffering from CLMs and insufficient FLRV volume. Its limitation is that it consists a possible stimulation of CLMs growth. Therefore, liver surgery should be performed as soon as possible after optimal FLRV growth without further delay. 


\section{Conflicts of Interest}

The Authors have no conflicts of interest to declare.

\section{Authors' Contributions}

Treska Vladislav: Study design, operation procedures, writing of article. Bruha Jan: Operation procedures, collection and application of aHSC, sample collection. Liska Vaclav: Study design, operation procedures. Fichtl Jakub: Operation procedures, sampling collection, preparation of material for statistical processing. Prochazkova Kristyna: Sample collection, preparation of material for statistical analysis. Petrakova Tereza: Sample collection, preparation of material for statistical analysis. Hosek Petr: Statistical analysis.

\section{Acknowledgements}

This study was supported by the Charles University Research Fund (Progres Q 39).

\section{References}

1 Ferlay J, Soerjomataram I, Dikshit R, Eser S, Mathers C, Rebelo M, Parkin DM, Forman D and Bray F: Cancer incidence and mortality worldwide: sources, methods and major patterns in GLOBOCAN 2012. Int J Cancer 136: E359-386, 2015. PMID: 25220842. DOI: $10.1002 / \mathrm{ijc} .29210$

2 Vaz da Silva DG, Ribeiro HSC, Arra DASM, Torres SM, Diniz AL, Godoy AL, Farias IC, Costa Jr WL and Coimbra FJ: Predictors of long-term survival in patients with hepatic resection of colorectal metastases: Analysis of a Brazilian Cancer Center Cohort. J Surg Oncol 121: 893-900, 2020. PMID: 32153041. DOI: $10.1002 /$ jso.25893

3 Fukami Y, Kaneoka Y, Maeda A, Takayama Y, Onoe S and Isogai M: Simultaneous resection for colorectal cancer and synchronous liver metastases. Surgery Today 46: 176-182, 2016. PMID: 26007322. DOI: 10.1007/s00595-015-1188-1

4 Russolillo N, Ratti F, Vigano L, Langella S, Cipriani F, Aldrighetti $\mathrm{L}$ and Ferrero A: The influence of aging on hepatic regeneration and early outcome after portal vein occlusion: A case-control study. Ann Surg Oncol 22: 4046-4051, 2015. PMID: 25758189. DOI: $10.1245 / \mathrm{s} 10434-015-4478-3$

5 Yamashita S, Hasegawa K, Takahashi M, Arita J, Sakamoto Y, Aoki T, Sugawara Y and Kokudo N: Hobson's choice two-stage hepatectomy for multiple and bilobar colorectal liver metastases with portal vein embolization: report of two cases. Surgery Today 45: 511-516, 2015. PMID: 24943807. DOI: 10.1007/ s00595-014-0953-x

6 Treska V: Methods to increase future liver remnant volume in patients with primarily unresectable colorectal liver metastases: Current State and Future Perspectives. Anticancer Res 36: 20652072, 2016. PMID: 27127106.

7 Moris D and Pawlik TM: Personalized treatment in patients with colorectal liver metastases. J Surg Res 216: 26-29, 2017. PMID: 28807210. DOI: 10.1016/j.jss. 2017. 04.013

8 Kow AWC: Hepatic metastasis from colorectal cancer. J Gastrointest Oncol 10: 1274-1298. 2019. PMID: 31949948. DOI: $10.21037 /$ jgo.2019. 08.06
9 Dörr NM, Bartels M and Morgul MH: Current treatment of colorectal liver metastasis as a chronic disease. Anticancer Res 40: 1-7, 2020. PMID: 31949948. DOI: 10.21037/jgo.2019.08.06

10 Schlitt HJ, Hackl C and Lang SA: 'In-situ split' liver resection/ALPPS - Historical development and current practice. Visc Med 33: 408-412, 2017. PMID: 29344513. DOI: $10.1159 / 000479850$

11 Luz JHM, Gomes FV, Coimbra E, Costa NV and Bilhim T: Preoperative portal vein embolization in hepatic surgery: A review about the embolic materials and their effects on liver regeneration and outcome. Radiol Res Pract 21: 1-9, 2020. PMID: 32148959. DOI: 10.1155/2020/9295852

12 Imai K, Adam R and Baba H: How to increase the resectability of initially unresectable colorectal liver metastases: A surgical perspective. Ann Gastroenterol Surg 3: 476-486, 2019. PMID: 31549007. DOI: 10.1002/ags3.12276

13 Albati NA, Korairi AA, Hasan IA, Almodhaiberi HK and Algarni AA: Outcomes of staged hepatectomies for liver malignancy. World J Hepatol 11: 513-521, 2019. PMID: 31293719. DOI: 10.4254/wjh.v11.i6.513

14 Huang HC, Bian J, Bai Y, Lu X, Xu YY, Sang XT and Zhao HT: Complete or partial split in associating liver partition and portal vein ligation for staged hepatectomy: A systematic review and meta-analysis. World J Gastroenterol 25: 6016-6024, 2019. PMID: 31660037. DOI: 10.3748/wjg.v25.i39.6016

15 Raptis DA, Linecker M, Kambakamba P, Tschuor C, Müller PC, Hadjittofi C, Stavrou GA, Fard-Aghaie MH, Tun-Abraham M, Ardiles V, Malagó M, Campos RR, Oldhafer KJ, HernandezAlejandro R, de Santibañes E, Machado MA, Petrowsky H and Clavien PA: Defining Benchmark Outcomes for ALPPS. Ann Surg 270: 835-841, 2019. PMID: 31592812. DOI: 10.1097/SLA. 0000000000003539

16 Niekamp AS, Huang SY, Mahvash A, Odisio BC, Ahrar K, Tzeng ChD and Vauthey JN: Hepatic vein embolization after portal vein embolization to induce additional liver hypertrophy in patients with metastatic colorectal carcinoma. Eur Radiol, 2020. PMID: 32144462. DOI: 10.1007/s00330-020-06746-4

17 Kobayashi K, Yamaguchi T, Denys A, Perron L, Halkic N, Demartines $\mathrm{N}$ and Melloul E: Liver venous deprivation compared to portal vein embolization to induce hypertrophy of the future liver remnant before major hepatectomy: A single center experience. Surgery 167: 917-923, 2020. PMID: 32014304. DOI: 10.1016/j.surg. 2019. 12.006

18 Guiu B, Quenet F, Escal L, Bibeau F, Piron L, Rouanet P, Fabre JM , Jacquet E, Denys A, Kotzki PO, Verzilli D and Deshayes E: Extended liver venous deprivation before major hepatectomy induces marked and very rapid increase in future liver remnant function. Eur Radiol 27: 3343-3352, 2017. PMID: 28101681. DOI: $10.1007 / \mathrm{s} 00330-017-4744-9$

19 Treska V, Fichtl J, Ludvik J, Bruha J, Liska V, Treskova I, Kucera R, Topolcan O, Lysak D, Skalicky T and Ferda J: Portal vein embolization (PVE) versus PVE with haematopoietic stem cell application in patients with primarily non-resectable colorectal liver metastases. Anticancer Res 38: 5531-5537, 2018. PMID: 30194213, DOI: 10.21873/anticanres.12888

20 Bruha J, Treska V, Mirka H, Hosek P, Fichtl J, Skalicky T, Bajcurova K, Ludvik J, Duras P, Lysak D and Liska V: Growth of colorectal liver metastases is not accelerated by intraportal administration of stem cells after portal vein embolization. Rozhl Chir 98: 159-166, 2019. PMID: 31159549. 
21 Ironside N, Bell R, Bartlett A, McCall J, Powell J and Pandanaboyana S: Systematic review of perioperative and survival outcomes of liver resections with and without preoperative portal vein embolization for colorectal metastases. HPB (Oxford) 19: 559-566, 2017. PMID: 28438427. DOI: 10.1016/j.hpb.2017.03.003

22 Loes IM, Immervoll H, Sorbye H, Angelsen JH, Horn A, Knappskog $\mathrm{S}$ and Loning PE: Impact of KRAS, BRAF, PIK3CA, TP53 status and intraindividual mutation heterogeneity on outcome after liver resection for colorectal cancer metastases. Int J Cancer 139: 647-656, 2016. PMID: 26991344. DOI: 10.1002/ijc.30089
23 Scherman P, Syk I, Holmberg E, Naredi P and Rizell M: Influence of primary tumour and patient factors on survival in patients undergoing curative resection and treatment for liver metastases from colorectal cancer. BJS Open 4: 118-132, 2020. PMID: 32011815. DOI: 10.1002/bjs5.50237

Received June 7, 2020

Revised June 28, 2020

Accepted July 2, 2020 